\title{
Serum tartrate-resistant acid phosphatase $5 b$ activity as a prognostic marker of survival in breast cancer with bone metastasis
}

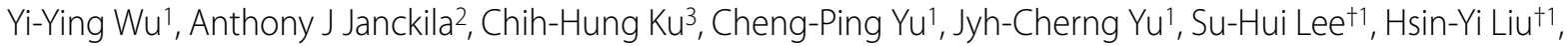 \\ Lung TYam² and Tsu-Yi Chao*1,4
}

\begin{abstract}
Background: Serum tartrate-resistant acid phosphatase $5 b$ (TRACP 5b) activity is a marker of osteoclast number and is elevated in breast cancer (BC) patients with extensive bone metastasis, which might in turn reflect the tumour burden. We tested the hypothesis that baseline serum TRACP 5b activity and its interval change are potential prognostic markers of survival in BC patients with bone metastasis.

Methods: We analyzed the data from previous prospective studies. A total of 100 patients with newly diagnosed bone metastasis were included. Cox proportional regression model was used to evaluate the correlation between the overall survival time (OS) and baseline serum TRACP $5 \mathrm{~b}$ activity and its interval changes. The least significant change (LSC) of TRACP $5 b$ was calculated from data obtained from 15 patients with early BC.

Results: Estrogen receptor status (Hazard Ratio $(H R)=0.397 ; p=0.003)$ and visceral metastasis $(H R=0.492 ; p=0.0045)$ were significantly correlated with OS. The OS was significantly shorter in those patients with higher baseline TRACP $5 b$ activity based on a cut-off value to delineate the highest tertile ( $H R=3.524 ; p<0.0001)$. Further analysis demonstrated that among patients in the highest tertile, OS was significantly longer in those patients who had achieved a decrease of serum TRACP 5b activity greater than the LSC (38.59\%) ( $p=0.0015)$.

Conclusions: We found that TRACP $5 \mathrm{~b}$ activity and its interval change after treatment bore a prognostic role in BC patients with bone metastasis and a high baseline serum TRACP 5b activity. Further prospective phase II study is necessary to confirm these results.
\end{abstract}

\section{Background}

Breast cancer $(\mathrm{BC})$ is the most common malignancy in women. Once metastasis occurs, most patients become incurable. However, metastatic $\mathrm{BC}$ is a heterogeneous disease in which some women survive only a few weeks whereas others survive many years. Hormonal receptor status including estrogen receptor (ER) and progesterone receptor (PR) as well as human epidermal growth factor receptor 2 (HER2) have been shown to predict treatment response and survival [1]. Patients with metastasis only to bone survive longer than those with visceral metastasis [2]. Nevertheless, patients with bone metastasis still carry

*Correspondence: tsuyi@ndmctsgh.edu.tw

1 Breast Cancer Research Group, Tri-Service General Hospital, National Defense Medical Center, Taipei, Taiwan

† Contributed equally

Full list of author information is available at the end of the article heterogeneous outcomes, and the survival time might become shorter when skeletal-related events (SRE) such as pathological fractures, radiation to bones, spinal cord compression, hypercalcemia, and bone surgery occur to patients [3]. Biomarkers of bone metabolism are eagerly sought as a way to predict outcome in $\mathrm{BC}$ patients with bone metastasis [3-5]. In a recent post hoc analysis from a randomized phase III trial comparing zoledronic acid and pamidronate in patients with bone metastasis, Lipton et al. demonstrated that early normalization of elevated baseline urinary N-telopeptide of type 1 collagen (NTX) levels was associated with longer event-free and overall survival (OS) times in $\mathrm{BC}$ patients receiving zoledronic acid[6,7]. Our previous studies demonstrated that NTX and tartrate-resistant acid phosphatase $5 \mathrm{~b}$ (TRACP 5b) have similar clinical performance for the diagnosis of 
bone metastases and treatment monitoring [8]. Furthermore, preanalytical variables such as diurnal rhythm and feeding influence NTX and other collagen biomarkers more than TRACP $5 \mathrm{~b}$ activity [8]. In most clinical practices it is difficult to standardize the time of blood draw. Therefore TRACP 5b has certain advantages over collagen biomarkers for assessment of bone metabolism.

TRACP $5 \mathrm{~b}$ is an enzyme secreted by osteoclasts and its activity can be measured specifically in serum by immunoassay[9]. Serum TRACP $5 \mathrm{~b}$ activity is a marker of osteoclast number and becomes elevated in the serum of individuals with increased bone turnover rate such as pre-pubertal children and post-menopausal women [911]. We and other investigators have found that serum TRACP $5 \mathrm{~b}$ activity could become elevated in BC patients with bone metastasis because $80 \%$ of the bone metastasis lesions caused by $\mathrm{BC}$ are osteolytic in nature [12,13]. Furthermore elevated serum TRACP $5 \mathrm{~b}$ activity in BC patients is positively correlated with the extent of bone metastasis $[8,14]$. We have also demonstrated that serum TRACP $5 \mathrm{~b}$ activity can be potentially used as a marker to monitor treatment response in $\mathrm{BC}$ patients with bone metastasis and as an adjunct to bone scintigraphy for the diagnosis and follow-up of bone metastasis $[8,14]$. In the current study we hypothesize that an elevated serum TRACP 5b activity may reflect a higher tumor burden and further signify a worse outcome in BC patients with bone metastasis. Therefore we examine if baseline serum TRACP $5 \mathrm{~b}$ activity and its interval change after treatment can be used as prognostic markers for overall survival time in BC patients with bone metastasis. Confounding factors including age, tumor markers including carcinoembryonic antigen (CEA) and CA 15.3, ER, HER2 overexpression, and the presence of visceral metastasis were adjusted.

\section{Methods \\ Study population}

We analyzed retrospectively biochemical and clinical data from $100 \mathrm{BC}$ patients with newly diagnosed bone metastasis who had participated in our previous studies of serum TRACP $5 \mathrm{~b}$ activity in BC $[12,13]$. ER and HER2 were stained by immunohistochemistry on paraffinembedded tumor tissues. Expression levels were determined semi-quantitatively by a $\mathrm{BC}$ pathologist (CPY). Bone metastasis was diagnosed according to clinical symptoms, radiological images, and $99 \mathrm{mTc}$-hydroxymethylene-diphosphanonate whole-body bone scintigraphy. All patients underwent chemotherapy, hormonal therapy, radiotherapy, and/or bisphosphonates therapy as clinically indicated. Fifteen informed, consenting subjects participated as a control group to determine the least significant change (LSC) of serum TRACP $5 \mathrm{~b}$ activity. The control subjects were females aged 34 to 67 years with a median age of 50 . All were in early stages of $\mathrm{BC}$ with no distant metastasis and undergoing adjuvant treatment as clinically indicated. All studies were performed under the guidelines of the Helsinki Declaration and approved by the Human Subjects Protection Offices (IRB) of Tri-Service General Hospital.

\section{Serum TRACP 5b Activity Assay}

Serum from each patient had been collected and processed at diagnosis of bone metastasis and monthly thereafter for 6 months or until death as described in previous studies $[12,13]$. Osteoclastic TRACP 5 b activity was measured by an antigen capture immunoassay as previously reported $[8,14]$. The clinical specificity and sensitivity of this assay for both osteoporosis and extensive bone metastasis in BC have been reported previously $[14,15]$. The analytic precision was estimated as the mean percent coefficient of variation (\%CV) for duplicate measurements. The inter-assay error was determined by repetitive independent assay over a one-week period of aliquots of six sera ranging in activity from 2.54 to $9.37 \mu \mathrm{mol} / \mathrm{min} /$ L. The average CV was calculated to be $3.9 \%$. The intraassay error was determined by simultaneous assay of eight duplicates of five sera ranging in activity from 2.50 to $11.0 \mu \mathrm{mol} / \mathrm{min} / \mathrm{L}$; the average $\mathrm{CV}$ was calculated to be $5.1 \%$.

\section{Statistical Analyses}

All descriptive data are expressed as median (range). The dependent variable was OS in days or months. Cox proportional regression model (SAS 9.1.3) was used to assess the association of interest, as well to adjust the potential confounders of age, tumor marker (CEA, CA 15.3), ER and HER2 status, and visceral metastasis. To avoid co-linearity among the independent variables, co-linearity diagnostic analysis was done with the following criteria: tolerance $>0.4$ or variance inflation $<2.5$ and condition number $<10$. There was no co-linearity among independent variables. Patients were divided into 2 groups according to their scales of TRACP $5 \mathrm{~b}$ activities, i.e. the top $1 / 3$ and the bottom $2 / 3$. Student $t$ test and Chi-square test were used to assess the allocation between these two groups. The LSC is the minimum change of TRACP $5 \mathrm{~b}$ activity considered to be of biological significance at a $95 \%$ level of confidence, a level of confidence normally used in clinical practice. In this study, we retrieved data from 15 randomly selected early breast cancer patients from previous studies to calculate the LSC of serum TRACP 5b from monthly samples taken over a 4-month period. The percent change in TRACP $5 \mathrm{~b}$ was calculated according to the following formula: $\Delta=$ [(baseline data subsequent data)/baseline data] $\times 100 \%$. Cox proportional regression model was used to compare survival of patients in the highest tertile of baseline TRACP $5 \mathrm{~b}$ activ- 
ity to the remaining patients in the lower and middle tertiles. The maximal interval change within 6 months for serum TRACP $5 \mathrm{~b}$ activity was used to stratify patients into two groups according to whether the interval change was greater or less than the LSC. Cox proportional regression model was used to estimate the significance and the hazard ratio of each variable in the total cohort, those in the highest TRACP $5 \mathrm{~b}$ tertile, and those in the remaining group separately.

\section{Results}

\section{Patient Characteristics}

The demographics of the $100 \mathrm{BC}$ patients with bone metastasis are shown in Table 1. They all were women ranging in age from 30 to 78 years with a median age of 50. The median OS was 481 days. Fifty-seven percent of patients had concurrent visceral metastases on the initial diagnosis of bone metastasis. Sixty-three of the 100 patients were ER-positive, 28 ER-negative, and 9 with unknown ER status. Eighteen of these patients were HER2-positive, 57 HER2-negative, and 25 had unknown HER2 status.

\section{Least Significant Change of TRACP 5b}

The LSC of serum TRACP $5 \mathrm{~b}$ activity over time was calculated from 4 consecutive monthly samples from 15

\section{Table 1: Characteristics of the 100 breast cancer patients} with bone metastasis

\begin{tabular}{|c|c|c|}
\hline Female/male & & $100 / 0$ \\
\hline Median age & & $50(30-78)$ \\
\hline $\begin{array}{l}\text { Median time from initial diagnosis to } \\
\text { enrollment }\end{array}$ & & $1007(2-6801)$ \\
\hline Median overall survival time (Days) & & $481(1-4465)$ \\
\hline Median initial TRACP-5b activity (U/L) & & $\begin{array}{l}3.894(1.21- \\
24.245)\end{array}$ \\
\hline Median CEA (ng/ml) & & $3.5(1-8165)$ \\
\hline Median CA-153 (U/ml) & & $\begin{array}{l}42.15(6.59- \\
3040)\end{array}$ \\
\hline \multirow[t]{3}{*}{ ER } & positive & 63 \\
\hline & negative & 28 \\
\hline & unknown & 9 \\
\hline \multirow[t]{3}{*}{ Her-2/neu } & positive & 18 \\
\hline & negative & 57 \\
\hline & unknown & 25 \\
\hline Visceral metastasis present & & 57 \\
\hline Bone metastasis only & & 43 \\
\hline \multirow[t]{4}{*}{ Prior chemotherapy } & None & 18 \\
\hline & One line & 46 \\
\hline & $\geq 2$ lines & 23 \\
\hline & unknown & 13 \\
\hline
\end{tabular}

patients with early $\mathrm{BC}$ without distant metastasis. The LSC of TRACP $5 \mathrm{~b}$ was $38.59 \%$. This meant that a TRACP $5 \mathrm{~b}$ change at months 1 to 4 of at least $38.59 \%$ was required to have a $95 \%$ level of confidence that a biologically significant change had occurred. If this change was a decline in serum TRACP $5 \mathrm{~b}$ activity, it was considered to be a positive treatment response and clinical improvement.

\section{Serum TRACP $5 b$ and Overall Survival Time}

Table 2 summarizes the results of Cox proportion regression models assessing the OS as a function of baseline variables including age, ER status, CEA, CA15.3, HER2 status and visceral metastasis in addition to baseline serum TRACP 5b activity. The presence of visceral metastasis in addition to bone metastasis as a dichotomous variable (Hazard ratio $(\mathrm{HR})=0.492 ; p=0.0045$ ) correlated inversely with the OS. ER positivity as a dichotomous variable correlated positively with a longer survival $(\mathrm{HR}=0.397 ; p=0.003)$. No other continuous biomarkers showed significant correlation with the OS. We divided the 100 patients into 2 groups arbitrarily according to the scale of serum TRACP $5 \mathrm{~b}$ activity, i.e. those with the top $1 / 3$ baseline activities (TRACP $5 \mathrm{~b} \geq$ $5.736 \mathrm{U} / \mathrm{L}$ ) and the remainders. The demographics and allocation between these 2 groups are listed in Table 3 . The OS in patients with the highest one-third of serum TRACP $5 \mathrm{~b}$ activity was significantly longer $(\mathrm{HR}=3.524$; $\mathrm{p}<0.001)$ than for those with the lower two-thirds of serum TRACP 5b (Table 2, Figure 1). We further calculated the median baseline serum TRACP $5 \mathrm{~b}$ activities in different patient groups stratified by the OS. As shown in Figure 2, there was an inverse relationship between baseline serum TRACP $5 \mathrm{~b}$ activity and OS.

\section{Serum TRACP 5b Interval Changes and the Overall Survival Time}

In the 100 patients studied, 81 had available serial serum TRACP $5 \mathrm{~b}$ activity determinations. No subsequent TRACP $5 \mathrm{~b}$ data could be retrieved from the remaining 19 patients, due to either early death or loss to follow up. The maximal interval change between baseline and follow-up serum TRACP $5 \mathrm{~b}$ activities $\left(\Delta_{\max }\right.$ TRACP $\left.5 \mathrm{~b}\right)$ within 6 months of enrollment in each individual patient was calculated as [(baseline TRACP 5b activity - TRACP $5 \mathrm{~b}$ activity at maximal alteration)]/baseline TRACP $5 \mathrm{~b}$ activity $\times 100 \%$. The median time to the maximal change of TRACP $5 \mathrm{~b}$ activity was 2 month. When the 81 patients' data were analyzed together, there was no correlation between $\Delta_{\max }$ TRACP $5 \mathrm{~b}$ and the OS. However, when only patients with baseline serum TRACP $5 \mathrm{~b}$ activities in the highest tertile $(\geq 5.736 \mathrm{U} / \mathrm{L})$ were analyzed $(\mathrm{n}=27)$, $\Delta_{\max }$ TRACP $5 \mathrm{~b}$, stratified by LSC as a dichotomous variable, i.e. $\Delta_{\max }$ TRACP $5 \mathrm{~b}>$ or $\leq 38.59 \%$, significantly cor- 


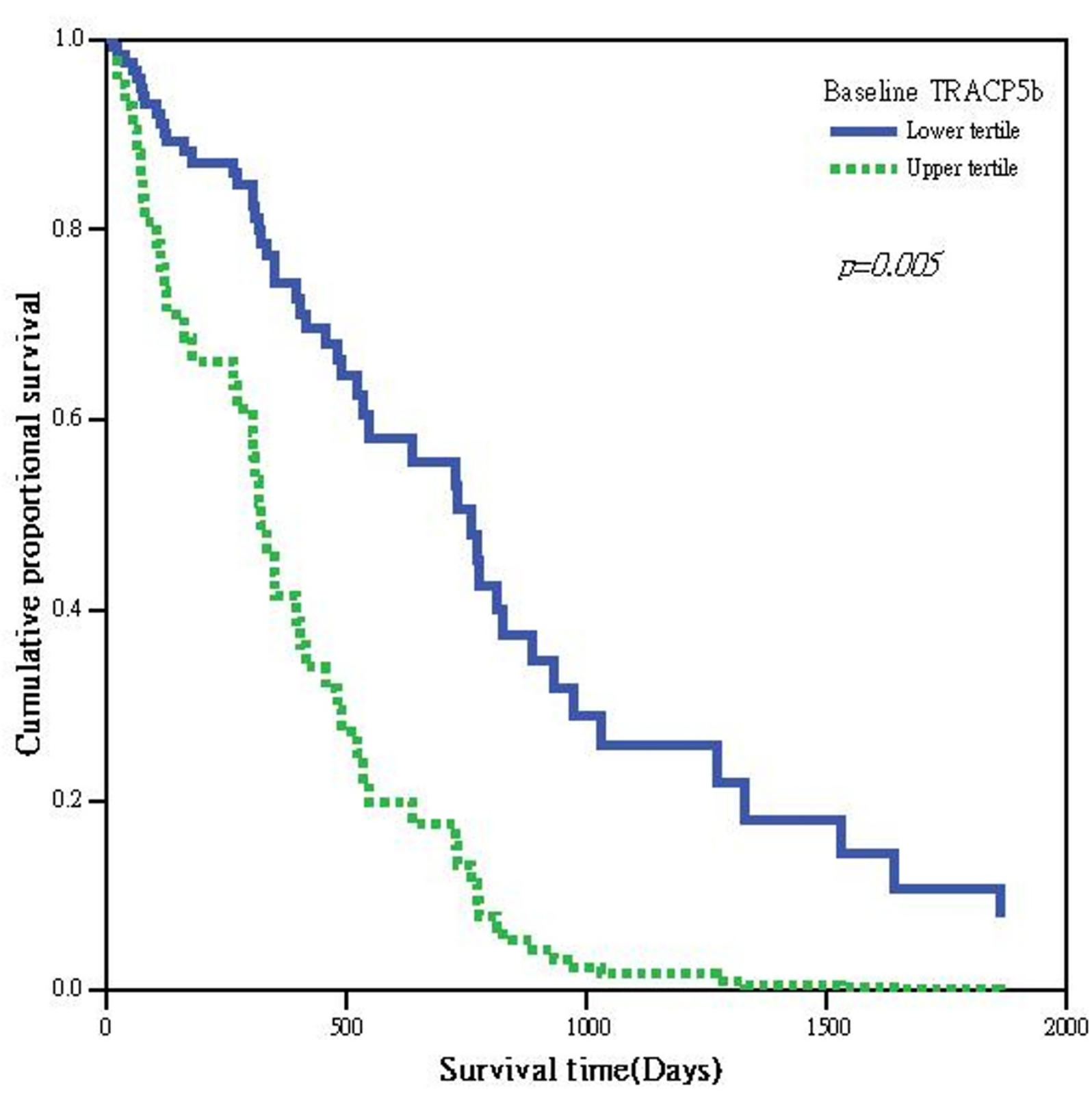

Figure 1 Survival curve comparing patients with baseline TRACP 5b activity in the upper third and the lower two thirds using Cox-regression model.

related with $\mathrm{OS}(\mathrm{HR}=0.213, p=0.0015$; Table 2 and Figure

\section{Discussion}

The survival of patients with metastatic $\mathrm{BC}$ varies from months to several years, and the most common metastatic site is bone. Metastasis to sites other than bone often dramatically shortens survival $[2,4]$. Bone metastasis is often related to ER-positivity and low grade tumor [16]. Our findings were consistent with previous studies in that ER positivity and bone-only were associated with a longer survival [17]. Tumor markers have been proven previously to have limited or no prognostic value $[2,18]$, and they were not prognostic markers for survival in this study. Specifically HER2 status did not contribute as a prognostic marker perhaps due to the use of trastuzumab in half of our patients.

When marrow is invaded by $\mathrm{BC}$ cells, osteoclasts are recruited and differentiated at an increased rate by the production of high amounts of tumor-derived cytokines 


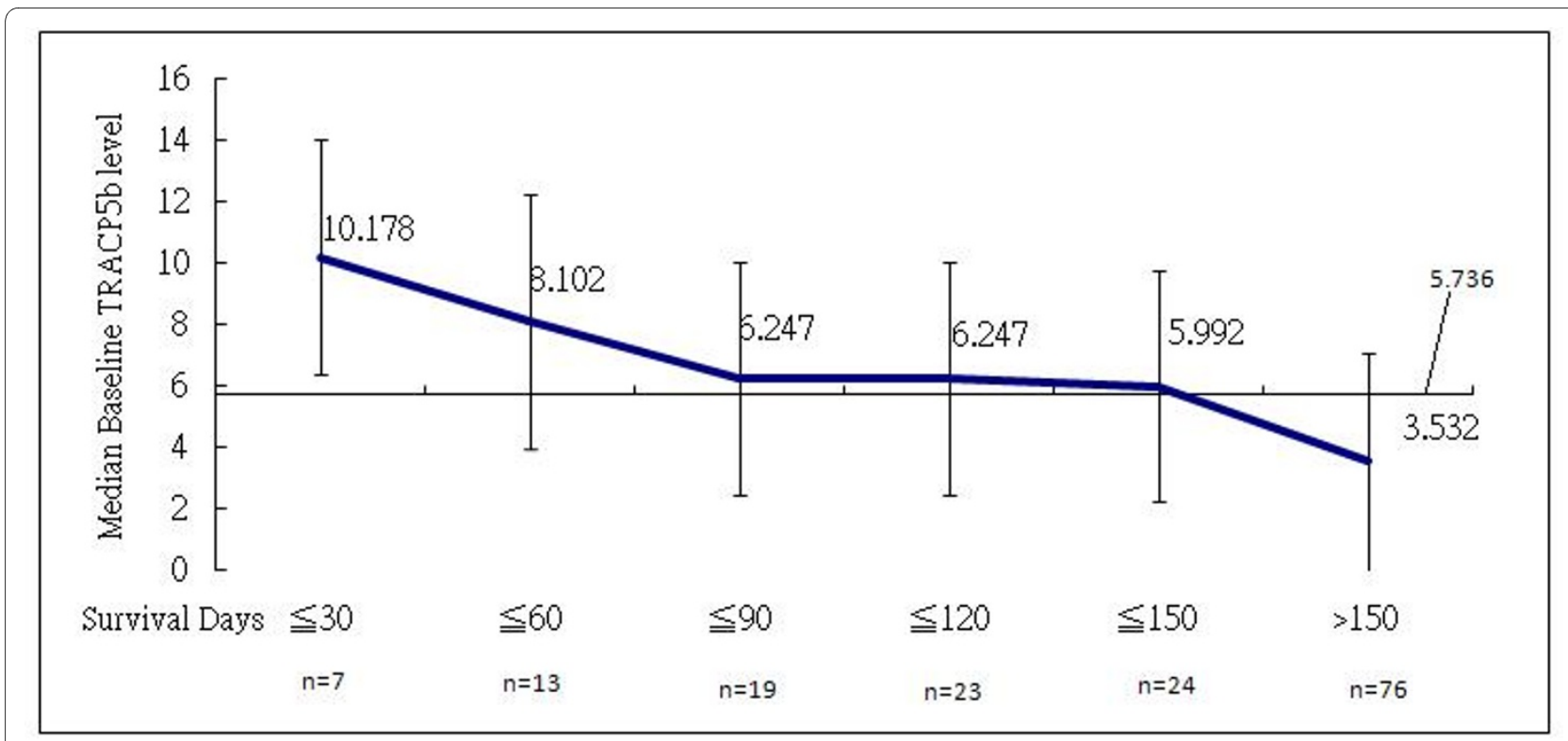

Figure 2 The median baseline TRACP $5 b$ activity $(U / L)$ in patients with different overall survival time

such as transforming growth factor $\beta$ (TGF- $\beta$ ), interleukin-6 (IL-6), parathyroid hormone-related protein
(PTHrP) [19]. It is reasonable to hypothesize that a higher tumor burden in the marrow could be associated with

Table 2: Cox-proportional regression analysis results of overall survival time of 100 breast cancer patients with bone metastasis.

\begin{tabular}{|c|c|c|c|c|c|c|c|c|c|c|c|c|}
\hline & \multirow{2}{*}{$\begin{array}{l}\text { Total } \\
\mathrm{HR}^{* *}\end{array}$} & cohort & \multicolumn{2}{|c|}{$n=100$} & Lower & $2 / 3^{*}$ & $n=54$ & & Top & $1 / 3^{*}$ & $\mathbf{n}=\mathbf{2 7}$ & \\
\hline & & $\mathrm{Cl}^{* *}$ & & $\mathbf{p}^{* * *}$ & HR & $\mathrm{Cl}$ & & $\mathbf{p}$ & HR & Cl & & $\mathbf{p}$ \\
\hline Age & $\mathrm{ns}^{* * * *}$ & - & - & ns & 0.945 & 0.896 & 0.997 & 0.0384 & ns & - & - & ns \\
\hline $\begin{array}{l}\text { Baseline TRACP } 5 b \\
\text { activity }\end{array}$ & 3.524 & 2.066 & 6.010 & $<0.001$ & - & - & - & - & - & - & - & - \\
\hline$\triangle \operatorname{maxTRACP} 5 b^{* * * * *}$ & - & - & - & - & ns & - & - & $\mathrm{ns}$ & 0.213 & 0.082 & 0.555 & 0.0015 \\
\hline CEA & $\mathrm{ns}$ & - & - & ns & ns & - & - & ns & ns & - & - & ns \\
\hline CA15.3 & ns & - & - & ns & ns & - & - & ns & ns & - & - & ns \\
\hline ER positivity & 0.397 & 0.240 & 0.656 & 0.0003 & 0.260 & 0.103 & 0.655 & 0.0043 & 0.374 & 0.148 & 0.947 & 0.0379 \\
\hline HER-2/neu positivity & ns & - & - & ns & ns & - & - & ns & ns & - & - & ns \\
\hline $\begin{array}{l}\text { Visceral metastasis } \\
\text { absent }\end{array}$ & 0.492 & 0.301 & 0.802 & 0.0045 & ns & - & - & ns & ns & - & - & ns \\
\hline
\end{tabular}


Table 3: Demographic and allocation analysis between highest tertile and lower tertiles

\begin{tabular}{|c|c|c|c|c|}
\hline & & $\begin{array}{c}\text { Top } 1 / 3 \\
(n=33) \\
(\text { Median } \pm \text { SD) }\end{array}$ & $\begin{array}{c}\text { Bottom 2/3 } \\
(n=67) \\
(\text { Median } \pm \text { SD) }\end{array}$ & p-value \\
\hline Age $^{*}$ & & $49.7 \pm 8.48$ & $50.0 \pm 9.29$ & 0.995 \\
\hline Survival time (days) * & & $202 \pm 264$ & $700 \pm 702$ & $<0.0001$ \\
\hline $\begin{array}{l}\text { Pre-enrolled time } \\
\text { (days) }{ }^{*}\end{array}$ & & $817 \pm 820$ & $1218 \pm 1584$ & 0.021 \\
\hline Baseline TRACP 5b* & & $8.006 \pm 3.921$ & $3.063 \pm 1.050$ & $<0.0001$ \\
\hline $\mathrm{CEA}^{*}$ & & $5.34 \pm 83.02$ & $2.50 \pm 1102$ & 0.577 \\
\hline CA $15.3^{*}$ & & $196.7 \pm 648.06$ & $32.59 \pm 142.42$ & $<0.0001$ \\
\hline \multirow[t]{2}{*}{ Visceral metastasis ${ }^{* *}$} & Positive & 22 & 35 & 0.248 \\
\hline & Negative & 11 & 32 & \\
\hline \multirow[t]{2}{*}{$E R^{* *}$} & Positive & 16 & 47 & 0.156 \\
\hline & Negative & 12 & 16 & \\
\hline \multirow[t]{2}{*}{$\mathrm{HER}^{* *}$} & Positive & 6 & 12 & 1.00 \\
\hline & Negative & 19 & 38 & \\
\hline \multirow[t]{3}{*}{ Prior chemotherapy ** } & None & 4 & 14 & 0.619 \\
\hline & One line & 16 & 30 & \\
\hline & $\geq 2$ lines & 7 & 16 & \\
\hline
\end{tabular}

higher cytokine levels, which could further result in increased numbers of mature osteoclasts and elevated serum TRACP $5 \mathrm{~b}$ activity.

We have already shown that serum TRACP 5 b activity correlates with extent of bone metastasis in $\mathrm{BC}$ patients [13]. Since a higher tumor burden is related to a poorer survival, we tested the hypothesis that elevated serum TRACP 5b activity, as a measure of extent of bone disease, could be a marker of poorer prognosis in $\mathrm{BC}$ patients with bone metastasis. In our study, TRACP $5 \mathrm{~b}$ activity was a significant prognostic marker for survival along with ER status and bone-only metastasis.

Nevertheless, serum TRACP $5 \mathrm{~b}$ activity may not be elevated in all $\mathrm{BC}$ patients with bone metastasis, only in those with extensive metastasis [14]. Previous receiver operative characteristic curve analysis showed that, although serum TRACP 5b activity is highly specific for bone metastasis, its sensitivity is only $80 \%$ [12]. Other factors to weaken this association could be whether bone metastasis is osteoblastic, osteolytic or mixed. If the lesions were primarily osteoblastic, serum TRACP 5b activity might be lower than those with osteolytic or mixed lesions (Personal observations by TYC). Baseline bone metabolic rate of individual patients may also confound analysis because age and the presence of osteoporosis have been shown to have impact on serum TRACP $5 \mathrm{~b}$ activity [12]. For example, serum TRACP 5b activity may be lower in pre-menopausal patients with bone metastasis. Although we corrected for age, $\mathrm{BC}$ and bone metastasis afflicts younger woman in Asia than in the West. Also in this regard, many post-menopausal women take anti-resorptive drugs to preserve bone health, which may cause apoptosis of osteoclasts and decrease the serum TRACP $5 \mathrm{~b}$ activity [10]. All these confounding factors may have weakened the power of serum TRACP $5 \mathrm{~b}$ activity in reflecting the tumor burden. In fact, some patients with a lower serum TRACP 5 b activity may actually carry a high tumor burden.

Accordingly, we proposed that only in those patients with higher serum activity can TRACP $5 \mathrm{~b}$ genuinely reflected higher tumor burden. Whereas in those patients with lower activities, serum TRACP $5 \mathrm{~b}$ may not be useful as a prognostic factor. However, the real cutoff value to differentiate high from low serum TRACP $5 \mathrm{~b}$ activity is unknown. Therefore we divided our patients into subgroups arbitrarily by the scale of baseline TRACP $5 \mathrm{~b}$ activities, i.e. top $1 / 3$ versus remaining $2 / 3$ with the cutoff value of $5.736 \mathrm{U} / \mathrm{L}$. We indeed found that those patients with baseline serum TRACP $5 \mathrm{~b}$ activity higher than 5.736 $\mathrm{U} / \mathrm{L}$ had a significantly shorter survival than the remainders.

Interval changes of bone markers after treatment in BC patients with bone metastases have been proposed as potential prognostic factors. Lipton et al. reported that a 


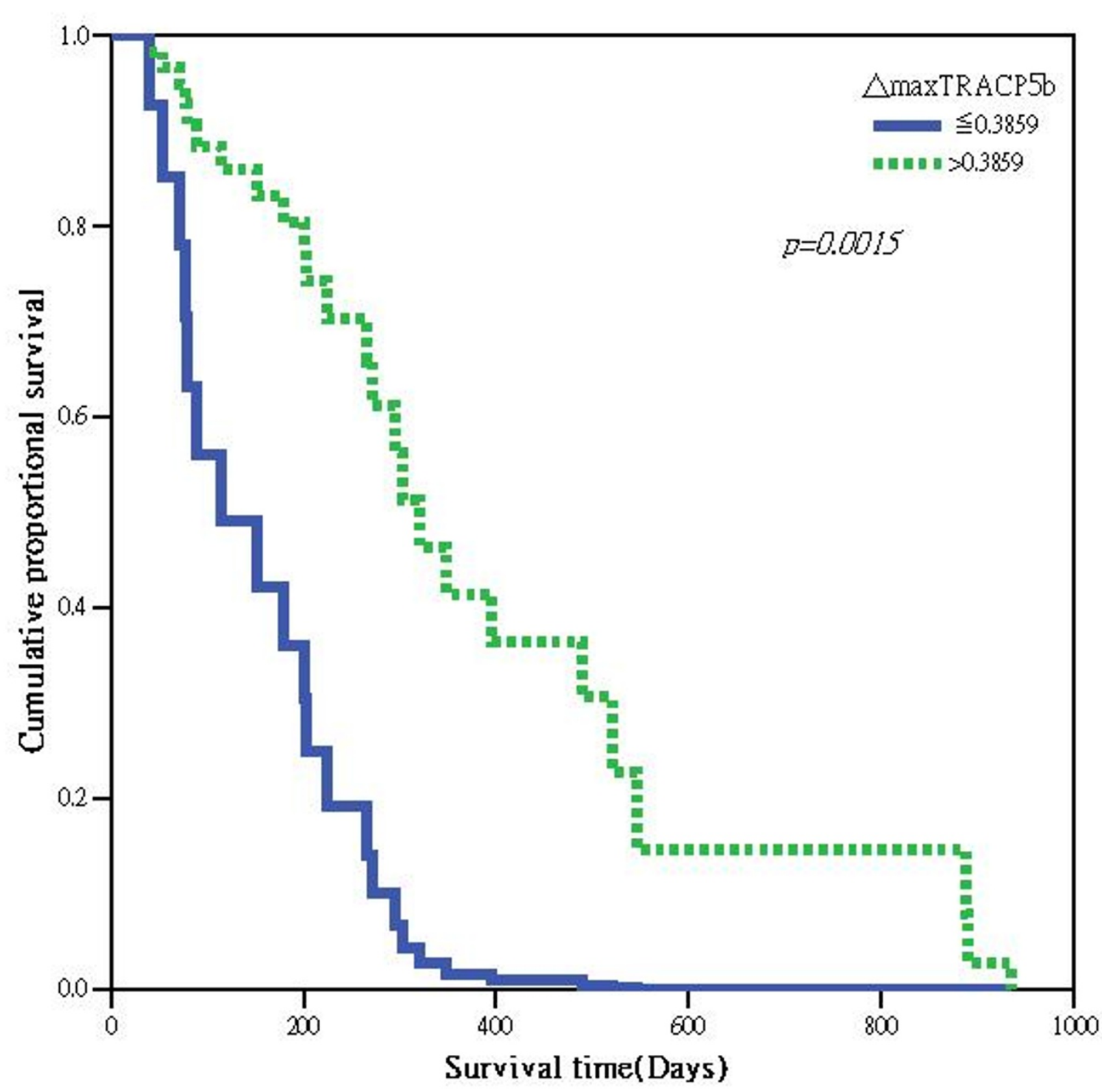

Figure 3 Survival curve comparing patients with baseline TRACP $5 \mathrm{~b}$ activity $\geq 5.736 \mathrm{U} / \mathrm{L}$ grouped by $\Delta_{\max }$ TRACP $5 \mathrm{~b}>38.59 \%$ and $\Delta_{\max }$ TRACP $5 \mathrm{~b} \leq 38.59 \%$ in a Cox-regression model.

persistently elevated urinary NTX level was related to poorer prognosis [6,7]. Early normalization of baseline elevated NTX was associated with longer event-free and overall survival times. Additionally, an extremely elevated baseline urinary NTX was associated with increased tumor burden in bone and an extremely aggressive disease. Our results showed that serum TRACP $5 \mathrm{~b}$ activity interval change might also be a potential prognostic marker, but it needs to be validated independently. In those patients with baseline serum TRACP $5 \mathrm{~b}$ activities higher than $5.736 \mathrm{U} / \mathrm{L}$, a $\Delta_{\max }$ TRACP $5 \mathrm{~b}$ greater than LSC after treatment had significantly longer OS compared to the rest of the group after adjustment of other prognostic factors. Serum TRACP $5 \mathrm{~b}$ activity of more than $5.736 \mathrm{U} / \mathrm{L}$ is probably related to a significant volume of cancer cells. Furthermore, when interval change of a biological marker is considered to be of prognostic value for a specific clinical setting, the LSC of a marker should be taken into account while interpreting the results. In our study, the LSC for serum TRACP $5 b$ activity was 
$38.59 \%$ determined from 15 early BC patients. When we dichotomized those patients whose serum TRACP $5 \mathrm{~b}$ activities were higher than $5.736 \mathrm{U} / \mathrm{L}$ into 2 groups based on response, we were able to demonstrate that patients with a $\Delta_{\max }$ TRACP $5 \mathrm{~b}>38.59 \%$ had a significantly longer survival than those without. However, this correlation was not existent when all patients were considered together. Other confounding factors such as ER status and bone-only metastasis correlated more strongly with the OS. $\Delta_{\max }$ TRACP $5 \mathrm{~b}$ lost its statistical significance as a prognostic marker for survival in the lower tertile groups. Our explanation is that serum TRACP $5 \mathrm{~b}$ may not reflect the real tumor burden in those patients with lower activities. This may have biased the result when all patients were included. Another possible explanation might be that $\Delta_{\max }$ TRACP $5 \mathrm{~b}$ only correlated with treatment response, which was not a strong prognostic marker for OS.

There are several limitations of this study. Firstly this is a retrospective study and patients did not receive uniform treatment. Nevertheless, it does reflect the real context of clinical practice, and effects due to specific treatments were not our primary objective. Secondly, the patient numbers are relatively small and the results could not be interpreted by stratification. Thirdly, tissues were not adequate enough or not available for determining ER status in 9 patients and HER2 in 25 patients. Fourthly, the cut-off value of $5.736 \mathrm{U} / \mathrm{L}$ was arbitrarily determined by dividing patients into top $1 / 3$ and bottom $2 / 3$ according to the scale of serum TRACP $5 \mathrm{~b}$ activity. This cut-off may not be the same in different laboratories and with different assays. Fifthly, 19 of the 100 patients in this study did not have serial TRACP $5 \mathrm{~b}$ data to calculate the association between its interval change and the overall survival time. This may have biased the final results.

\section{Conclusion}

Our study, deemed as a concept proving study, has shed light on the relative diagnostic and prognostic value of serum TRACP $5 \mathrm{~b}$ in $\mathrm{BC}$ patients with bone metastasis. The research effort is exploratory and hypothesis-generating only, given the small sample size and certain study design limitations. To determine the true value of TRACP $5 \mathrm{~b}$ as a prognostic marker for all patients with bone metastasis will require a prospective phase II study over a longer period including more patients to allow for more refined stratification.

\section{Competing interests}

The authors declare that they have no competing interests.

\section{Authors' contributions}

YYW analyzed the clinical information along with laboratory data and drafted the manuscript. CHK performed the statistical analysis. CPY reviewed the pathological slides. TYC designed and oversaw the progress of the study as well as finalized the manuscript. JCY and LTY gave criticisms to this manuscript. AJ set up the TRACP 5b immunoassay, critically reviewed and assisted in writing this manuscript. SHL and HYL carried out the biomarker immunoassays and were responsible for the quality assurance. All authors read and approved the final manuscript.

\section{Acknowledgements}

This study was supported by a grant issued from the National Science Council of Taiwan (NSC 95-2314-B-016-039-MY3). The authors would like to thank LiLing Huang, and San-Yu Lin of the Division of Hematology/Oncology, Department of Medicine, Tri-Service General Hospital, for their assistance of data collection and summarization.

\section{Author Details}

'Breast Cancer Research Group, Tri-Service General Hospital, National Defense Medical Center, Taipei, Taiwan, 2Division of Hematology, Veterans

Administrative Medical center, Louisville, Kentucky, USA, ${ }^{3}$ Department of Public Health, National Defense Medical Center, Taipei, Taiwan and ${ }^{4}$ National Institute of Cancer Research, National Health Research Institutes, Taipei, Taiwan

Received: 30 July 2009 Accepted: 23 April 2010

Published: 23 April 2010

\section{References}

1. Brown M, Tsodikov A, Bauer KR, Parise CA, Caggiano V: The role of human epidermal growth factor receptor 2 in the survival of women with estrogen and progesterone receptor-negative, invasive breast cancer: the California Cancer Registry, 1999-2004. Cancer 2008, 112(4):737-747.

2. James JJ, Evans AJ, Pinder SE, Gutteridge E, Cheung KL, Chan S, Robertson JF: Bone metastases from breast carcinoma: histopathological radiological correlations and prognostic features. Br J Cancer 2003, 89(4):660-665

3. Coleman R, Brown J, Terpos E, Lipton A, Smith MR, Cook R, Major P: Bone markers and their prognostic value in metastatic bone disease: Clinical evidence and future directions. Cancer Treat Rev 2008, 34(7):629-639.

4. Coleman RE: Clinical features of metastatic bone disease and risk of skeletal morbidity. Clin Cancer Res 2006, 12 (20 Pt 2):6243s-6249s.

5. Voorzanger-Rousselot N, Juillet F, Mareau E, Zimmermann J, Kalebic T, Garnero P: Association of 12 serum biochemical markers of angiogenesis, tumour invasion and bone turnover with bone metastases from breast cancer: a crossectional and longitudinal evaluation. Br J Cancer 2006, 95(4):506-514.

6. Lipton A, Cook RJ, Major P, Smith MR, Coleman RE: Zoledronic acid and survival in breast cancer patients with bone metastases and elevated markers of osteoclast activity. Oncologist 2007, 12(9):1035-1043.

7. Lipton A, Cook R, Saad F, Major P, Garnero P, Terpos E, Brown JE, Coleman RE: Normalization of bone markers is associated with improved survival in patients with bone metastases from solid tumors and elevated bone resorption receiving zoledronic acid. Cancer 2008, 113(1):193-201.

8. Chung YC, Ku CH, Chao TY, Yu JC, Chen MM, Lee SH: Tartrate-resistant acid phosphatase $5 \mathrm{~b}$ activity is a useful bone marker for monitoring bone metastases in breast cancer patients after treatment. Cancer Epidemiol Biomarkers Prev 2006, 15(3):424-428.

9. Halleen JM, Alatalo SL, Suominen H, Cheng S, Janckila AJ, Vaananen HK: Tartrate-resistant acid phosphatase $5 \mathrm{~b}$ : a novel serum marker of bone resorption. J Bone Miner Res 2000, 15(7):1337-1345

10. Nenonen A, Cheng S, Ivaska KK, Alatalo SL, Lehtimaki T, Schmidt-Gayk H, Uusi-Rasi K, Heinonen A, Kannus P, Sievanen $\mathrm{H}$, et al: Serum TRACP $5 \mathrm{~b}$ Is a Useful Marker for Monitoring Alendronate Treatment: Comparison With Other Markers of Bone Turnover. J Bone Miner Res 2005, 20(8):1804-1812

11. Chen CJ, Chao TY, Janckila AJ, Cheng SN, Ku CH, Chu DM: Evaluation of the activity of tartrate-resistant acid phosphatase isoform $5 \mathrm{~b}$ in normal Chinese children--a novel marker for bone growth. J Pediatr Endocrinol Metab 2005, 18(1):55-62

12. Chao TY, Yu JC, Ku CH, Chen MM, Lee SH, Janckila AJ, Yam LT: Tartrateresistant acid phosphatase $5 \mathrm{~b}$ is a useful serum marker for extensive bone metastasis in breast cancer patients. Clin Cancer Res 2005, 11 (2 Pt 1):544-550. 
13. Chao TY, Ho CL, Lee SH, Chen MM, Janckila A, Yam LT: Tartrate-resistant acid phosphatase $5 \mathrm{~b}$ as a serum marker of bone metastasis in breast cancer patients. J Biomed Sci 2004, 11(4):511-516.

14. Tsai SH, Chen CY, Ku CH, Janckila AJ, Yam LT, Yu JC, Chuang KW, Chao TY: The semiquantitative bone scintigraphy index correlates with serum tartrate-resistant acid phosphatase activity in breast cancer patients with bone metastasis. Mayo Clin Proc 2007, 82(8):917-926.

15. Halleen JM, Ylipahkala H, Alatalo SL, Janckila AJ, Heikkinen JE, Suominen $H$, Cheng S, Vaananen HK: Serum tartrate-resistant acid phosphatase $5 b$, but not $5 a$, correlates with other markers of bone turnover and bone mineral density. Calcif Tissue Int 2002, 71(1):20-25.

16. Wei $B$, Wang J, Bourne $P$, Yang $Q$, Hicks D, Bu H, Tang P: Bone metastasis is strongly associated estrogen receptor-positive/progesterone receptornegative breast carcinomas. Hum Pathol 2008, 39(12):1809-1815.

17. Maki HS, Hoehn JL: Influence of estrogen receptors on survival and recurrence in patients with breast cancer without lymph node metastases. Arch Surg 1989, 124(3):377-380.

18. Tsukushi S, Katagiri H, Kataoka T, Nishida Y, Ishiguro N: Serum tumor markers in skeletal metastasis. Jpn J Clin Oncol 2006, 36(7):439-444.

19. Chu P, Chao TY, Lin YF, Janckila AJ, Yam LT: Correlation between histomorphometric parameters of bone resorption and serum type $5 \mathrm{~b}$ tartrate-resistant acid phosphatase in uremic patients on maintenance hemodialysis. Am J Kidney Dis 2003, 41(5):1052-1059.

\section{Pre-publication history}

The pre-publication history for this paper can be accessed here: http://www.biomedcentral.com/1471-2407/10/158/prepub

doi: 10.1186/1471-2407-10-158

Cite this article as: Wu et al., Serum tartrate-resistant acid phosphatase $5 b$ activity as a prognostic marker of survival in breast cancer with bone metastasis BMC Cancer 2010, 10:158

Submit your next manuscript to BioMed Centra and take full advantage of:

- Convenient online submission

- Thorough peer review

- No space constraints or color figure charges

- Immediate publication on acceptance

- Inclusion in PubMed, CAS, Scopus and Google Scholar

- Research which is freely available for redistribution 